\title{
Research on Interactive Medical Kit Based on the Needs of People with Visual Impairment
}

\author{
Zhao Zhanfeng \\ Wenzhou Vocational \& Technical College, Wenzhou. 325035 \\ Zhanfeng@qq.com
}

Keywords: Visual Impairment, Convenience, Interaction, Medical Kit.

\begin{abstract}
It is dangerous to forget to take medicine, repeat to take and have fainting at home or just it is not convenient to find medicine, therefore a product has reminding function is very necessary. From this aspect, it becomes my starting point for design. The product is designed to have simple look and inside, the design is reasonable and humanized. With new material adopted, it can realize the man-machine interaction dedicated for the elders.
\end{abstract}

\section{Usability of Medical Kit}

Briefly speaking, the usability means that the product is easy to be used or not, or how easy to be used. Based on the man-machine design and psychology, usability serves as one of the requirements of product design and also it is the demand for use preferences today. The product with such property is mostly called as "friendly" product. It acts as a solution for the issue whether the user can properly use the product. In Product International Standardization Organization (ISO), this property is defined that the effect, efficiency and customer satisfaction of product used by specific user to realize specific goals in a specific environment. The purpose of product usability is to make product close to user so that they can learn how to use it more convenient (Fig.3). The usability of a medical Kit covers four aspects:

\section{Design Plan}

The final modeling of the kit is determined under practical specific requirements in the way to meet the use of the old. It has clear modules and elegant proportions. From its appearance, it is designed to provide the old with a dependable psychological suggestion and the color should be reasonable. In terms of the development of society, under modern process, it uses new materials and has multiple functions. The product put its actual value into the space utility and economic benefit. In addition, its appearance expresses deep modern scientific and technological spirit and the purchase of green design. In this design, the medical kit for home use has natural curves and graceful gradually varied surface. As a whole, it provides a comfortable visual perception for customer, as shown in Fig.1: 


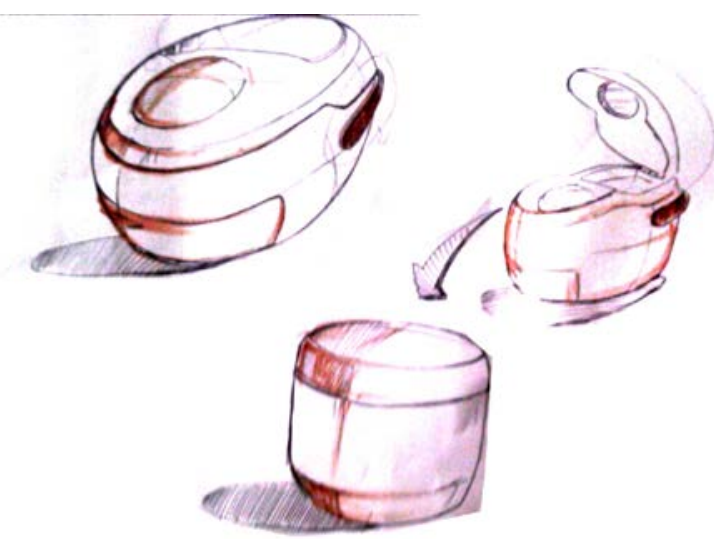

Fig.1 Draft

For the inside details, the original tip is adopted and a collecting unit is added in the grater to ensure the vegetable is clean and sanitary, reducing additional work that the user will collect extra vegetables after working. The scientific technology is used in the tip selected and use. The complicated internal structure compare to the simple outer modeling, inspiring people's curiosity, as shown in Fig.2.

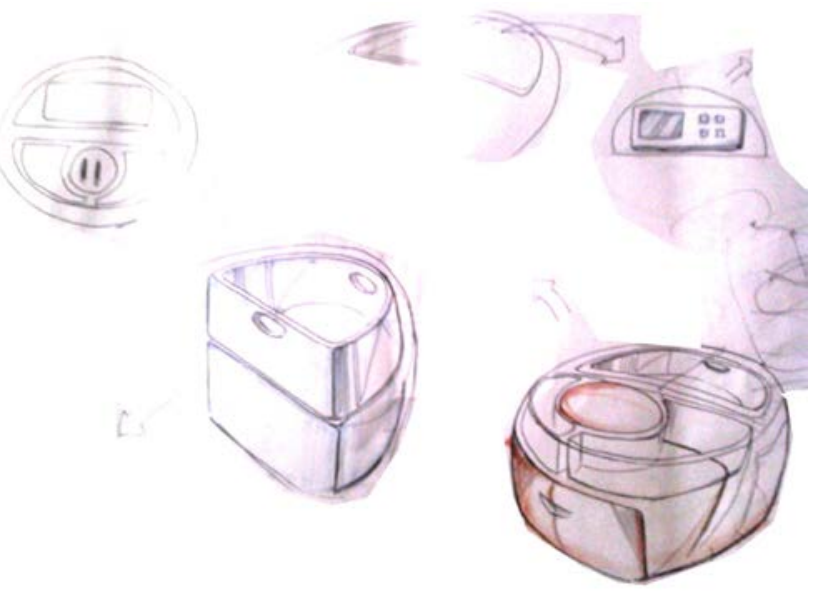

Fig. 2 Details

\section{The 3D Effect of Product}

The product is a breakthrough of normal modeling, and it brings a sense of sureness for the old. Furthermore, it also reduce psychological stress or passive thinking like "I am old". The handle is designed at the middle-back position of the whole body to be taken conveniently. A main control panel is located in the middle part of cover, providing various functions such as voice prompt, time setting, information scanning and screen display etc., as shown in Fig.3. 


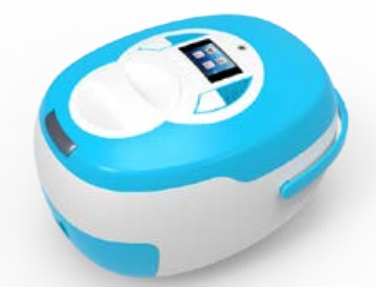

Fig. 3 Effect Picture

\section{Product Modeling}

Modeling, the image of an object, is a kind of visual language expressed with lines as main symbols in object form. The modeling element is an important part to be considered in design concerns. The nature and features of designs should be defined, specific and tangible through a certain modeling. The design of medical kit modeling is implemented on the separation and combination of modeling components such as point, line, face, body, color and texture. In order to endow these elements acceptable specific aesthetic significance, designers will use some concrete modeling forms like unity and change, symmetry and balance, rhythm and rhyme. In this complex procedure, the key factor is to make each element to reach a maximum coordination. In design field, the unification of form and functions is explored forever. The two are closely connected, function affects forms and vice versa. Product position determines that both form and function are at same level.it is not hard to find that successful design product has distinct form, together with enlightening and educational functions. In the modeling, the function design is added to meet the needs of the old.

\subsection{Product Structure}

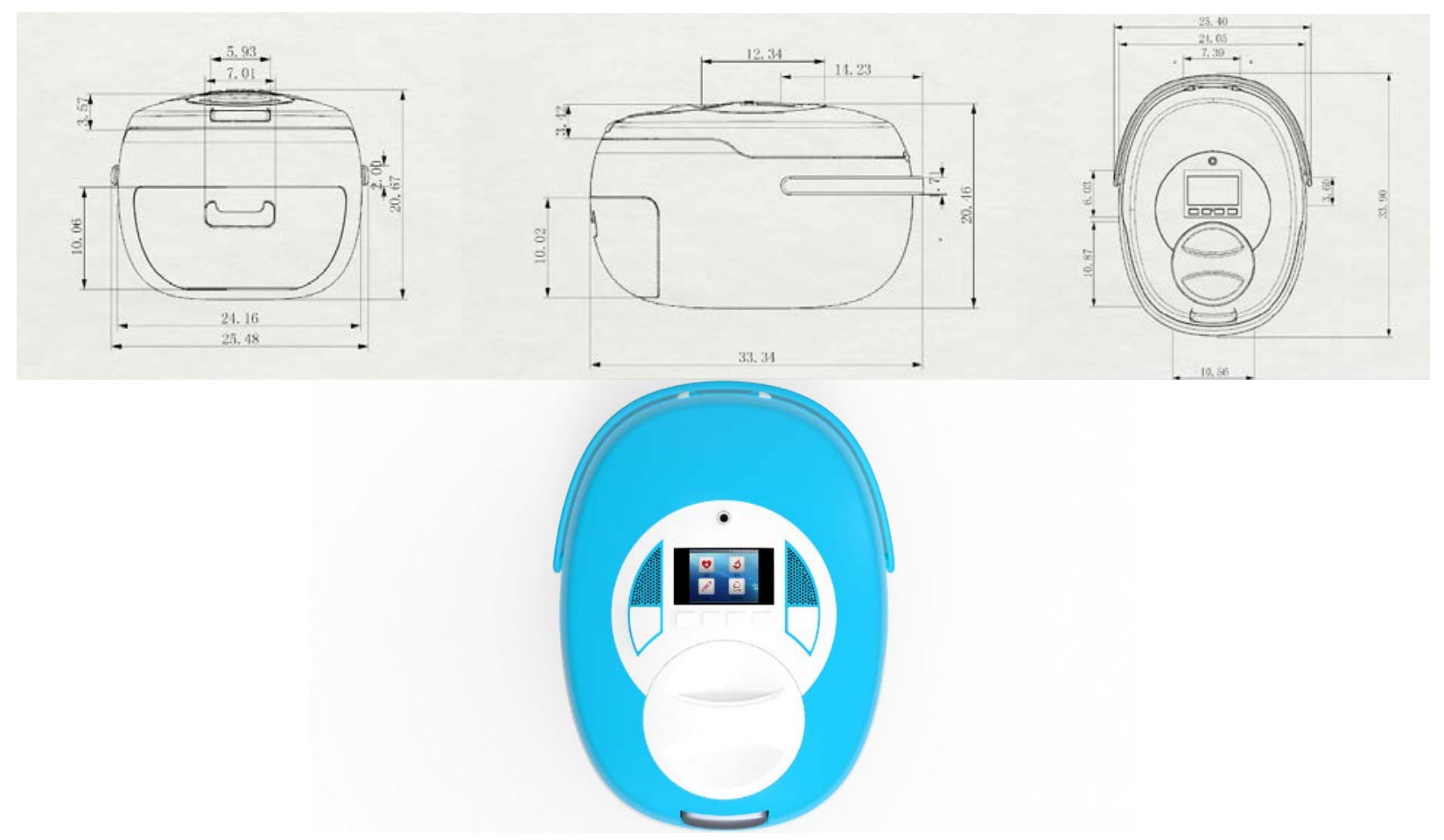

Fig.4 Dimensional Drawing 
After studying current products in market, we defines that the size of the product is 33.4*25.5*24(cm), as shown in Fig.4.

\subsection{Analysis on Product Appearance Modeling}

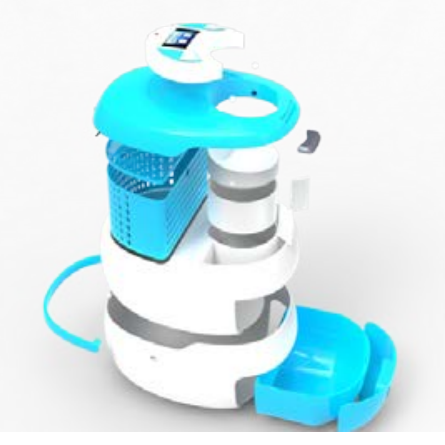

Fig.5 Explosive View

From the top to bottom, they are main control panel, cover of kit, switch, cover of small box, small box, plastic interlayer, small plastic frame, main body, shell, handle, small drawer, shell of drawer, as shown in Fig.5.

\subsection{Product Detail Drawing Display}

It can realize function that time setting for medicine taking is done and there will be voice prompt. Press buttons to control switch or setting. The scanning unit can help the old to identify the medicine type, avoiding the possibility of taking the wrong medicine. The button under loudspeaker is for One Touch SOS to avoid the panicky occurrence when disease comes on at night, in such case, the old can call for help immediately only by pressing the button. The top is a scanner. The old has bad eyesight that they cannot read relevant handwriting clearly. The scanner will help to display all content and provide corresponding voice prompt only by scanning the medicine.

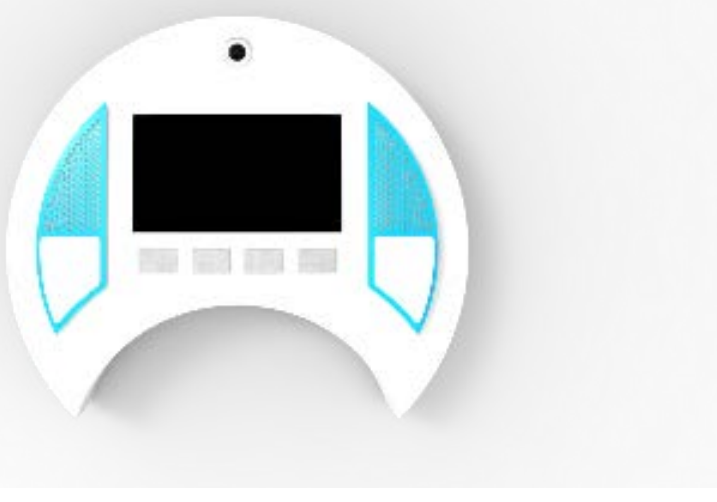

Fig.6 Panel

The most front is a carry-on small box. You can unscrew it in rotatory mode and remove cover to take medicine inside of the box. The details are as shown in Fig.7. 


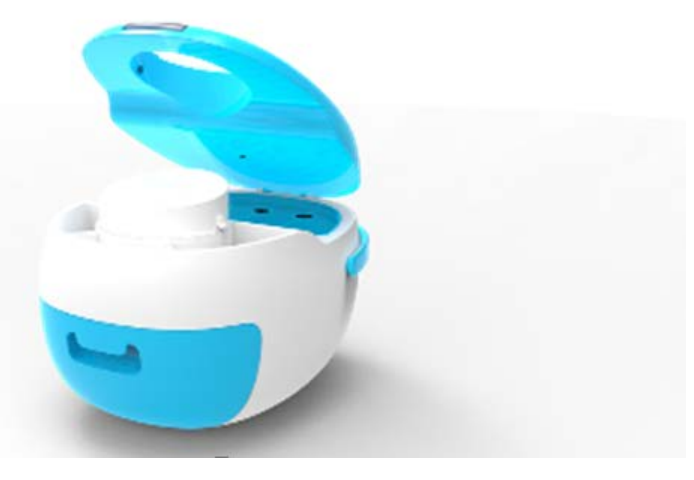

Fig.7 Cover Status

The blue plastic part is a storage box to collect small stuff like small scissors and band-aids. They can be taken out to use at any time. If medicine packed in a bag which is hard to tear, then the small scissors can be used, as shown in Fig. 8.

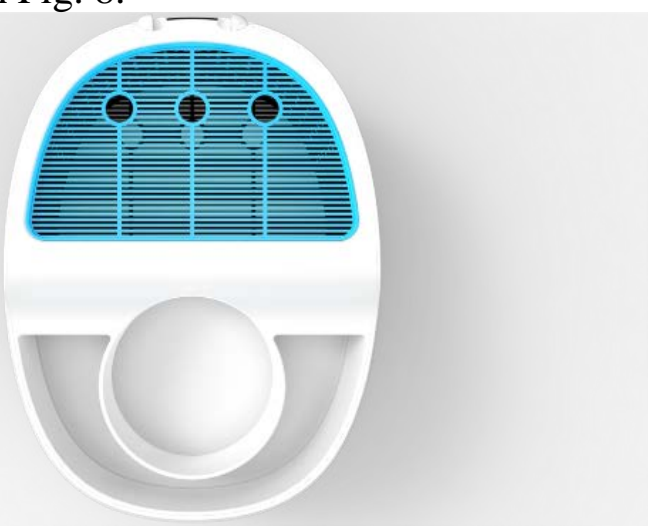

Fig.8 Easy to use

Taking out the interlayer, you will find a large space to place some medicines which are not commonly used. In the large space, some other things can be put here at the same time, as shown in Fig.9.

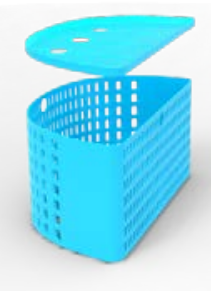

Fig.9 Interlayer

The handle at the middle is for easy taking, which is simple and convenient for the old. Another one is located in the rear part of the product, keeping the color and modeling matching that of the whole product, as shown in Fig.10. 


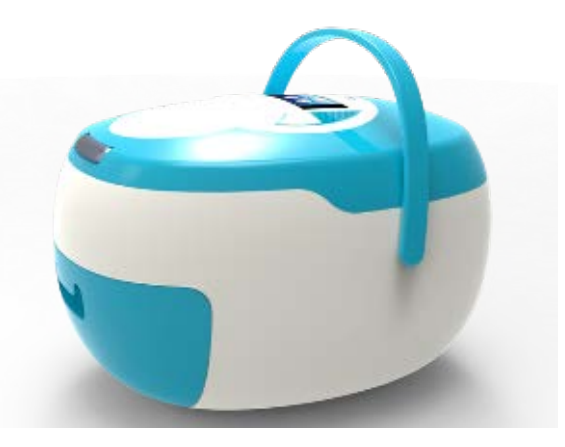

Fig.10 Carrying Type

Unlike common medical kit, this kit only need to pull gently instead of exerting your strength to press, then all medicines will show in small drawers. Some commonly used medicines in big packages can also be put in and you can arrange them as your needs, as shown in Fig.11.

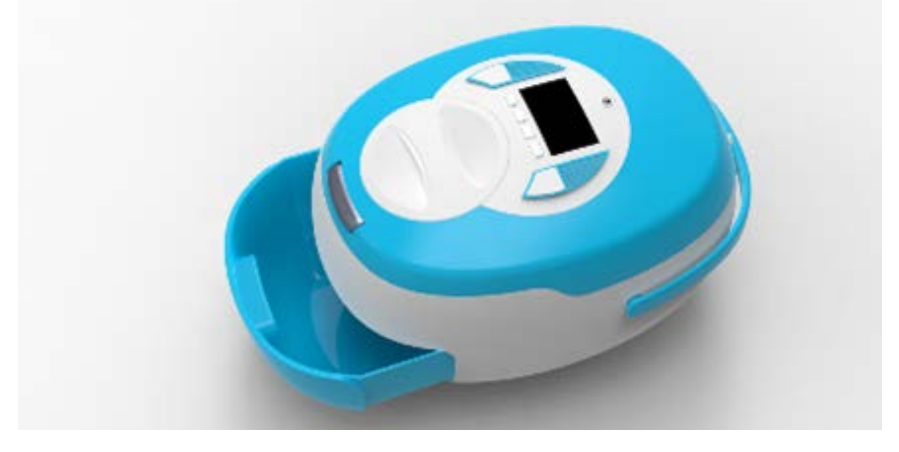

Fig. 11 Pulling Type

\section{Product Material}

The performance characteristics of material are fully used to improve product quality or meet some new functions requirements, so that people's life quality can be further improved. Therefore, to develop and apply new material in design is important part to improve product effect utility and develop new product functions. With the advance of scientific technology, the material to make medical kit is increasing. In our days, the textures are widely applied in design and production of medical kit including all plastics, all metal, aluminum plastic, wooden etc. The main materials of shell of the medical kit are zein plastics and metal material, highlighting the inside shadow and color change.

\section{Analysis on Product Material Process}

Poly-lactic acid particles extracted from maize are called "zein plastics", or scientific name of "poly-lactic acid", and it can replace chemical plastic particles. So the selection of material is "zine plastic”. At present, such material has entered industrialized trial production stage. This material, a bioenvironmental material that can be completely degraded, can fully replace chemical plastic. Therefore, it is regarded as the fourth new material following metal material, inorganic material, and high molecular material. The product made from zine plastic has mild color and softer hand feeling compared to that of plastic products. 


\section{Product Structure}

The structure design of the kit mainly reflects the reasonable distribution of modules inside the kit. Firstly, considering the features of the old, it is necessary to analyze the multiple storage spaces and the medicines status (such as solid or liquid). Secondly, the internal structure of the kit shall be handled according to different packages of medicines to arrange big, middle and small spaces. Last, the function, man-machine engineering, design psychology also needs to be carefully considered and improved, as shown in Fig.12.

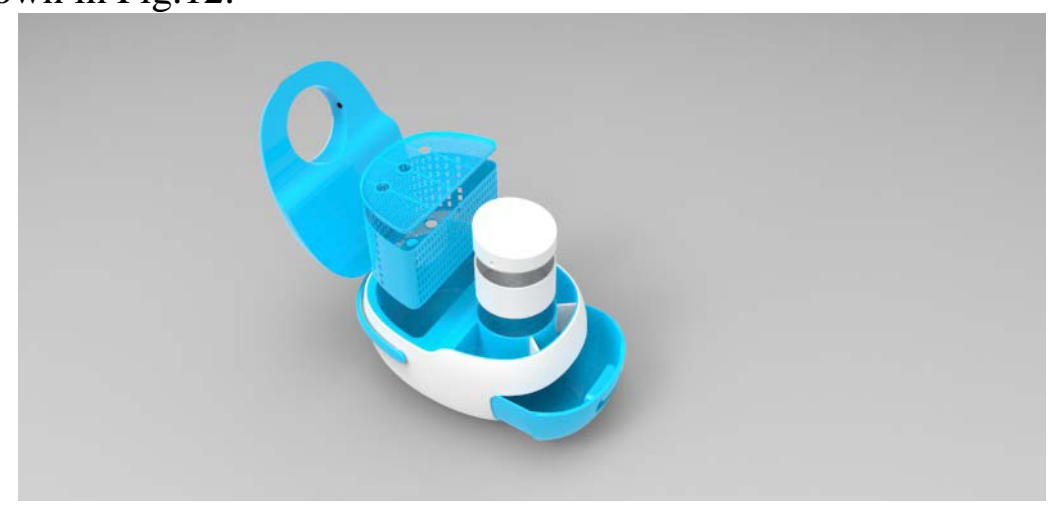

Fig.12 Reasonable Distribution

\section{Product Functions}

In our memory, medical kit has always been playing a storage role. In this design, various additional functions are discussed and innovated without limitation. By using various high technologies, the medical kit for home use meets the physiological needs of older people and provides more and better functions. This design focuses on the structure of medical kit and using modern scientific technologies.

\section{Application of Ergonomics in Medical Kit Design}

In this design of medical kit for home use, the man-machine design is expressed on the man-machine interaction, life style of older people and physiologic habits etc. For example, the button design (designed as rotary button), humanization and fluency of operating panel to solve the bad eyesight of the old. The color scheme is shown in Fig.13.
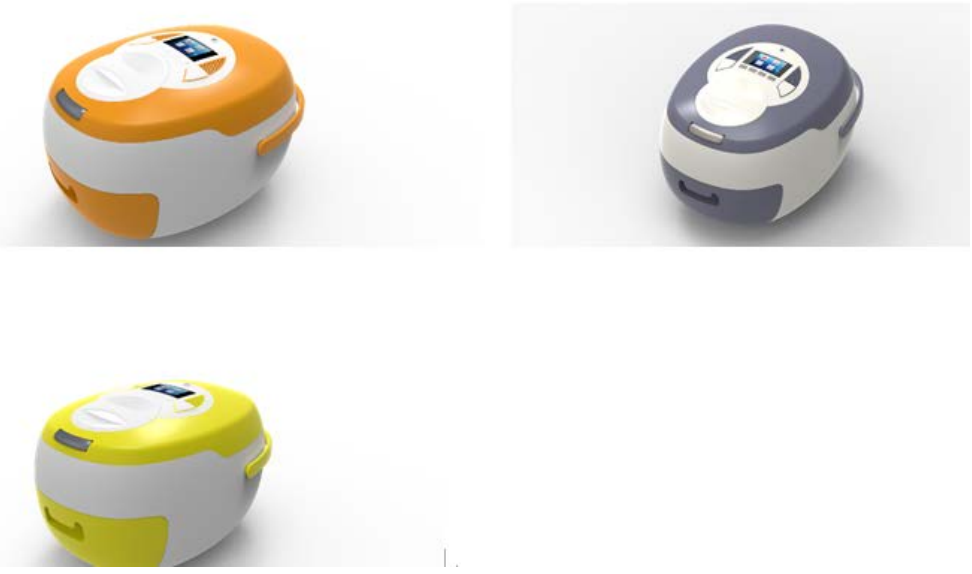

Fig.13 Color 


\section{Conclusion}

The usability of product is determined by product and the user. The different physiological structures of people lead to different physical sizes of product. As a result, the realization of usability of product is defined by ergonomics and product semantics. Therefore, the designer should, based on the principle of simplifying complicated, avoid burdensome design as much as possible.

\section{References}

[1]Christian Boris Brunner,Sebastian Ullrich,Patrik Jungen,Franz-Rudolf Esch. Impact of symbolic product design on brand evaluations[J]. Journal of Product \& Brand Management,2016,25(3).

[2]Anders Warell. Identity References in Product Design: An Approach for Inter-relating Visual Product Experience and Brand Value Representation[J]. Procedia Manufacturing,2015,3.

[3]Nathanael Ko,Roberta Graf,Tom Buchert,Marcus Kim,Daniel Wehner. Resource Optimized Product Design Assessment of a Product's Life Cycle Resource Efficiency by Combining LCA and PLM in the Product Development[J]. Procedia CIRP,2016,57.

[4]David Peck,Prabhu Kandachar,Erik Tempelman. Critical materials from a product design perspective[J]. Materials and Design,2015,65.

[5]Rafiqul Gani,Ka M. Ng. Product design - Molecules, devices, functional products, and formulated products[J]. Computers and Chemical Engineering,2015,81.

[6]Minu Kumar,Charles H. Noble. Beyond form and function: Why do consumers value product design?[J]. Journal of Business Research,2016,69(2).

[7]KwanMyung Kim. Collaborative product design processes of industrial design and engineering design in consumer product companies[J]. Design Studies,2016,46.

[8]Chung-Yang Chen,Gen-Yih Liao,Ku-Shen Lin. An attribute-based and object-oriented approach with system implementation for change impact analysis in variant product design[J]. Computer-Aided Design,2015,62.

[9]Qing WU. Traditional Chinese culture in modern Product Design[J]. Canadian Social Science,2015,11(10).

[10]Shih-Wen Hsiao,Cheng-Ju Tsai. Transforming the natural colors of an image into product design: A computer-aided color planning system based on fuzzy pattern recognition[J]. Color Research \& Application,2015,40(6).

[11]Marzena E. Nieroda,Mona Mrad,Michael R. Solomon. How do consumers think about hybrid products? Computer wearables have an identity problem[J]. Journal of Business Research,2018,89.

[12]Albert Albers,Jonas Heimicke,Benjamin Walter,Gustav Nils Basedow,Nicolas Reiß,Nicolas Heitger,Sascha Ott,Nikola Bursac. Product Profiles: Modelling customer benefits as a foundation to bring inventions to innovations[J]. Procedia CIRP,2018,70.

[13]Jing Li,Yafei Nie,Xinwei Zhang,Keqin Wang,Shurong Tong,Benoît Eynard. A Framework Method of User-participation Configuration Design for Complex Products[J]. Procedia CIRP,2018,70.

[14]Mary Kathryn Thompson,Ida Kirstine Juel Jespersen,Thomas Kjœrgaard. Design for manufacturing and assembly key performance indicators to support high-speed product development[J]. Procedia CIRP,2018,70.

[15]Shu-you Zhang,Ye Gu,Xiao-jian Liu,Jian-rong Tan. A knowledge push technology based on applicable probability matching and multidimensional context driving $[J]$. Frontiers of Information Technology \& Electronic Engineering,2018,19(2). 